\title{
BENEFICIOS Y PROBLEMÁTICAS EN LA APLICACIÓN DE NORMAS INTERNACIONALES DE AUDITORÍA EN MÉXICO
}

\author{
BENEFIC AND PROBLEMS IN THE APLICATION OF THE \\ INTERNATIONALS RULES OF AUDIT IN MEXICO \\ Dorie Cruz Ramírez* \\ Suly Sendy Pérez Castañeda** \\ Pilar Heriberto Moreno Uribe*** \\ Universidad Autónoma del Estado de Hidalgo/ Escuela Superior de Cd. Sahagún / México \\ [Recepción: Julio de 2013/ Conformidad: Agosto 2013]
}

\section{RESUMEN}

La adopción de las NIA para México, así como, para otros países del mundo, presentan beneficios y problemáticas que son consideradas como áreas de oportunidad. Dicha normatividad es considerada una norma mundial, con mayor grado de rigurosidad y universalidad, que pretende contribuir a la estabilidad financiera mundial y contrarrestar la volatilidad que acompaña a la globalización, pero también requiere un diagnóstico sobre regulaciones y legislaciones del país, para que a su vez, permitan la implementación total de estas normas. Las NIA en la actualidad, otorga a la auditoría, mayor confianza en la normatividad mexicana, dado que se sustenta bajo normas de confiabilidad y certeza, de que los estados financieros generados por las empresas están normados de manera uniforme, lo que trae por consecuencia lógica mayor aceptación de los datos que contienen, por lo tanto, permiten el cotejo de información financiera en un ambiente internacional, lo que conlleva a que cada vez genere mayor promoción de la inversión extranjera en el país, en una palabra la armonización del ámbito contable y de auditoría a nivel internacional, ya que hablamos de que se cuenta con una metodología estructurada para el correcto desarrollo de la auditoría.

Palabras Claves: Información financiera, auditoría y Normas Internacionales de Auditoría.

\begin{abstract}
The adoption of the ISAs, to Mexico and other countries, has benefits and problems that are seen as areas of opportunity, such a regulation is considered a worldwide standard, with a greater degree of rigor and universality, which aims to contribute to the global financial stability and offset the volatility that accompanies globalization, but also requires an assessment of regulations and legislation in the country to turn, allow the full implementation of these standards. The NIA currently given to the audit, greater confidence in Mexican law, since it sustains bajonormas reliability and certainty, that the financial statements generated by companies, are regulated uniformly, which brings logical greater acceptance of the data they contain, therefore, allow the comparison of financial reporting in an international environment, which in turn leads each generates more promotion of foreign investment in the country, in a word the harmonization of accounting field audit and internationally, and we talk to has a structured methodology for the correct development of the audit.
\end{abstract}

Keywords: Financial Reporting, Auditing and International Standards on Auditing.

\footnotetext{
* Maestra en Gestión Administrativa, Especialista en Impuestos, Licenciada en Contaduría. Email: doriec@uaeh.edu.mx

** Maestra en Administración de Instituciones, Licenciada en Contaduría y Finanzas, Licenciada en Ciencias de la Comunicación. Email: sulysp@uaeh.edu.mx.

*** Doctor en Ciencias Administrativas, Maestro en Administración Tributaria, Especialista en Impuestos, Licenciado en Contaduría. Email: hmoreno_uribe@hotmail.com
} 


\section{METODOLOGÍA}

Investigación exploratoria y descriptiva, debido a que su objetivo radica en la obtención de un conocimiento y la realización de un breve análisis sobre conceptos propios de auditoría, como son información financiera, conceptualizaciones de auditoría, y sus normatividades, bajo la perspectiva de una investigación documental, de artículos realizados por expertos en la materia.

\section{INTRODUCCIÓN}

Este tema es de vital interés, para quienes hacen uso de la auditoría, tanto profesionales auditores como empresas obligadas o no a auditarse, ya que es importante conocer que beneficios y problemáticas se pueden encontrar en la implementación de las NIA.

En los últimos años y debido a los procesos de globalización de los procesos contables, los contadores que se desarrollan preponderantemente en el área de la auditoría, se encuentran ocupados con distintas exigencias para el desarrollo de dicha actividad, por mencionar algunas de ellas se tiene, la adecuación de Normas de Auditoría Generalmente Aceptadas (NAGA) a las Normas Internacionales de Auditoría (NIA), la aplicación de normas de control de calidad para las firmas que están vigentes a partir de 2012, dado que las revisiones cada vez son más exhaustivas, así como, las exigencias de los usuarios de la información financiera, entre otras tantas más.

Para Moreno (2012), la labor de un contador consiste en generar información oportuna, veraz y confiable, y es por ello, que dicha regla no exime a los auditores, ya que a través de sus exámenes tienen acceso a elementos vitales en las organizaciones, y la primicia de toda auditoría es emitir una opinión sobre la razonabilidad de los estados financieros; así mismo, el auditor se allega de elementos aislados que con su experiencia convierte en información importante para la organización y para todo aquel interesado.

Es por ello, que resulta de interés realizar un análisis sobre la incorporación de las normas internacionales de auditoría a las aplicables en México y bajo qué perspectivas se da dicha incorporación.

\section{GENERALIDADES DELAAUDITORÍA SOBRE INFORMACIÓN FINANCIERA}

Para Hurtado (2013), auditoría es el examen profesional, objetivo e independiente de las operaciones financieras y/o administrativas, que se realizan con posterioridad a su ejecución, tanto en empresas públicas como privadas y cuyo producto final es un informe que muestre la razonabilidad de la situación de la empresa; así mismo, donde se establezca una opinión sobre la información auditada, tanto como conclusiones y recomendaciones, encaminadas a promover la economía, eficiencia y cumplimiento de las leyes y regulaciones aplicables de dicha entidad.

Así también, la auditoría es considerada un instrumento de gestión, y evaluación sistemática, documentada y objetiva, para lo cual debe ser realizada de acuerdo a normatividades previamente establecidas. Cabe hacer mención, que el propósito que persigue una auditoría, consiste en incrementar el grado de confianza de los diversos usuarios en los estados financieros. Y esto se logra con la emisión de una opinión por parte de un auditor, sobre si los estados financieros están elaborados en todos los aspectos importantes, de acuerdo con un marco de referencia de información financiera aplicable.

Es importante mencionar que el marco de referencia para auditoría, consiste en formular una opinión sobre si los estados financieros están presentados, razonablemente respecto de todo lo importante, o dan un punto de vista verdadero y razonable. Una auditoría conducida de acuerdo con la normatividad vigente, (NIA), Normas Internacionales de Auditoría, y los requisitos éticos relevantes, posibilita al auditor a formarse esa opinión.

Por lo tanto, los estados financieros sujetos a auditoría, son aquellos que son elaborados por la administración de la entidad. Una auditoría, de acuerdo a la normatividad vigente, se conduce sobre la premisa de que la administración prepara la información acorde dichas normas establecidas, y bajo ciertas responsabilidades que son fundamentales para la realización de la auditoría. Es importante mencionar que una auditoría de los estados financieros, no releva a la 
administración de sus responsabilidades. Pero partimos con el cuestionamiento sobre ¿Cuáles son las normatividades establecidas que den cumplimiento a la formulación de una auditoría?

\section{NORMAS DE AUDITORÍA}

Para dar cumplimiento al cuestionamiento anterior, como primer punto se analiza qué son las Normas de Auditoría en México conocidas hasta este momento y para Hurtado (2013), menciona que éstas son lineamientos que en forma obligatoria los auditores tienen que cumplir en el desempeño de sus actividades de auditoría y presentan los requisitos personales y profesionales del auditor, las orientaciones para la uniformidad en el trabajo con el propósito de lograr un buen nivel de calidad en el examen, así como lo concerniente a la elaboración del informe de auditoría.

Estas normas fueron emitidas en 1955 por la Comisión de Normas y Procedimientos de Auditoría (CONPA), cabe hacer mención que dichas normas, constituyeron las bases para construir el prestigio de la confiabilidad y honestidad de la profesión contable en México, (principalmente en el área de auditoría), y otros países, durante un periodo aproximado de 57 años.

Debido a los procesos actuales de globalización y la importancia que tiene el poseer información financiera oportuna, correcta y confiable, que genera tomas de decisiones de trascendencia. Por lo tanto, se requiere que los estándares de confiabilidad estén normados de manera uniforme y consistente al mundo actual en el que vivimos. De ahí entonces, la importancia que las normas adquieran carácter internacional.

\section{NORMAS INTERNACIONALES DE AUDITORÍA}

Con lo antes establecido, todos los trabajos de auditoría de información financiera sobre los ejercicios que iniciaron a partir del 1 de enero de 2012, deben ser realizados bajo los lineamientos establecidos por las Normas Internacionales de Auditoría, (NIA) para
México y las ISA (sus siglas en inglés) que a su vez, son emitidas por el Consejo de Normas Internacionales de Auditoría y Aseguramiento (IAASB) de la Federación Internacional de Contadores (IFAC).

Con lo anterior expuesto, debe de quedar entendido que las normas de auditoría que anteriormente se empleaban deben ser suplidas por las Normas Internacionales de Auditoría.

Por su parte Cámara (2012), comenta que es importante mencionar que dicha adopción no incluye las Normas para atestiguar, Normas de revisión, las Normas para otros servicios relacionados, y Normas de control de calidad aplicable a las firmas de contadores públicos que desempeñan auditorías y revisiones de información financiera, trabajos para atestiguar y otros servicios relacionados, cuya emisión queda a cargo de la CONAA (Comisión de Normas de Auditoría y Aseguramiento).

Las Normas Internacionales de Auditoría (NIA) para México, quedan clasificadas de la siguiente forma:

- NIA 100-199 Asuntos Introductorios y marco de Referencia de las NIA.

- NIA 200-299 Principios generales y responsabilidades, se detallan los objetivos generales de la auditoría, las responsabilidades del auditor al realizar una auditoría siguiendo los preceptos de las ISA., incluye el requerimiento del acuerdo con los términos del trabajo de auditoría, el control de calidad en el trabajo y la documentación de la auditoría.

- NIA 300-399 Planeación, Conocimiento del negocio e importancia relativa de la auditoría. Proporciona las normas y guías necesarias para que el auditor pueda planear la auditoría partiendo de un conocimiento profundo de la entidad, su entorno.

- NIA 400-499 Control interno, Evaluación del riesgo y respuesta a los riesgos identificados. Incluye la evaluación del control interno para la preparación de la información financiera.

- NIA 500-599 Evidencia de auditoría. Establece los objetivos a ser alcanzados en el proceso de obtención de la evidencia de auditoría. 
- NIA 600-699. Utilización del trabajo de terceros. Describe los requisitos mínimos y los objetivos que se deben buscar cuando se usa el trabajo de terceros en el proceso de obtención de evidencia de auditoría.

- NIA 700-799 Conclusiones y dictamen de auditoría.

- NIA 800-899 Área especializada de auditoría.

- NIA 900-999 Servicios relacionados.

- NIA 1000-1100 Declaraciones Internacionales de auditoría.

Las NIA están elaboradas en el contexto de una auditoría de estados financieros. Estas se adaptarán según sea necesario, a las circunstancias cuando se apliquen a auditorías de otra información financiera histórica.

Como base para la opinión del auditor, las NIA requieren que el auditor obtenga una seguridad razonable sobre si los estados financieros como un todo están libres de alguna representación errónea de importancia relativa, ya sea por fraude o por error.

La aplicación de las NIA, otorga un alto nivel de seguridad razonable, y este nivel se seguridad se obtiene cuando el auditor ha obtenido suficiente evidencia apropiada de auditoría para reducir el riesgo de auditoría. Esta normatividad tiene bien establecido los objetivos, requisito para soportar la opinión del auditor.

Las NIA requieren que el auditor ejerza su juicio profesional y mantenga el escepticismo profesional durante la planeación y desempeño de la auditoría de estados financieros sujetos a examen.

\section{CONCLUSIONES:}

A manera de conclusión se puede establecer que :

1. La adopción de las NIA presenta beneficios positivos, como lo establece Gutiérrez (2013). Estas son:

-Mayor confiabilidad a nivel internacional sobre los resultados obtenidos de auditorías de estados financieros.

98/ QuipuramaYoc | Vol. 21(40) 2013
- Generación de mayor promoción de inversión extranjera en el país.

- La aplicación de metodologías y manuales de auditoría de la mayoría de las firmas de mayor renombre en el mundo, debido a que se encuentran ya alineadas a esta nueva normatividad.

- Mayor cotejo con el ambiente internacional de la información financiera.

- Mayor confianza en el esquema de normatividad mexicana.

- Generación de mayor promoción de inversión extranjera en el país.

- Apoya la armonización del ámbito contable a nivel internacional.

2. La aplicación de las NIA proporciona al auditor una metodología estructurada para el correcto desempeño de una auditoría basada en los principios como queda establecido en la NIA 200. Por otra parte, Drosdoff (2001) mencionó que la implementación de Normas Internacionales de Auditoría, al considerarse una norma mundial, al ser más rigurosa y universal en las naciones, contribuirá a la estabilidad financiera mundial y contrarrestará parte de la volatilidad que acompaña a la globalización. La adhesión a normatividades internacionales, es considerada una herramienta que puede conducir a reducir riesgos y redituar beneficios financieros y económicos. Se reduce la incertidumbre, esto se debe a que al contar con una normatividad globalizada se considera que existe mayor trasparencia sobre la información financiera.

3. Por lo tanto, tendríamos que preguntarnos ¿Las empresas mexicanas, están lo suficientemente preparadas para la adopción de las NIA?.

\section{RECOMENDACIONES}

Pero no todo son beneficios, los auditores en la aplicación de las NIA, se pueden enfrentar algunas problemáticas o desventajas que hay que tomar en consideración como son:

- Se requiere rediseñar los sistemas contables, debido a que serán analizados bajo una perspectiva diferente a los sistemas que se han venido generando a través de los últimos años. 
BENEFICIOS Y PROBLEMÁTICAS EN LA APLICACIÓN DE NORMAS INTERNACIONALES DE AUDITORIA EN MÉXICO

- Se requiere mayor control interno en las entidades para generar información financiera susceptible de ser evaluadas por auditoria aplicando las NIA.

- Aún falta divulgación y conocimiento sobre como es el funcionamiento de las NIA.

- Y algo de vital importancia, se deben de realizar previamente un diagnóstico sobre regulaciones y legislaciones del país, que permitan la implementación total de la normatividad internacional.

Por último, es importante mencionar que debido a los procesos de globalización y unificación del criterio contable las NIA se deben de aplicar de manera obligatoria.

\section{REFERENCIAS HEMEROGRÁFICAS}

1. CAMARA, L. (2012) Adopción de las normas: El camino hacia las ISA. Revista Veritas, Noviembre 2012, LVI No. 1695. CCPM. p.p. 26-27.

2. MORENO, S. (2012) El rumbo de la auditoría. Revista Veritas, Junio 2012, LVI No. 1690. CCPM.p.p. 20-21

\section{REFERENCIAS ELECTRÓNICAS}

1. DROSDOFF, D. (2001), Normas Internacionales de Contabilidad y Auditoría. Revista del Banco interamericano de Desarrollo. http:// www.docstoc.com/docs/115415127/Normasinternacionales-de-contabilidad-y-auditoria-unbuena (visitado el 07-04-2013).

2. GUTiÉRREZ, F. (2013) Normas Internacionales de Auditoria en México. Entre letras y números IMPC Vol. 22 Diciembre-Enero 2013 http://www.ccpm.org.mx/servicios/ gaceta_universitaria/diciembre_enero_2013/ art_tecnico1.html (visitado el 02-04-2013).

3. HURTADO, P. (2013) Curso elemental de auditoría. http://www.emagister.com/cursoelemental-auditoria/concepto-auditoria (visitado el 08-04-2013).

4. IMCP (2013) Normas internacionales de auditoría NIA 200. http://imcp.org.mx/wp-content/ uploads/2013/01/NIA_200.pdf (visitado el 01-04-2013). 